\title{
Anomalous flows in a sunspot penumbra
}

\author{
Rohan E. Louis ${ }^{1}$, Christian Beck ${ }^{2}$, Shibu K. Mathew ${ }^{3}$, and P. Venkatakrishnan ${ }^{3}$ \\ ${ }^{1}$ Leibniz-Institut für Astrophysik Potsdam (AIP), An der Sternwarte 16, 14482 Potsdam, Germany \\ e-mail: rlouis@aip.de \\ 2 National Solar Observatory, Sacramento Peak, 3010 Coronal Loop, Sunspot, New Mexico 88349, USA \\ ${ }^{3}$ Udaipur Solar Observatory, Physical Research Laboratory, Dewali, Badi Road, 313004 Udaipur, Rajasthan, India
}

Received 1 May 2014 / Accepted 27 August 2014

\begin{abstract}
Context. The photospheric Evershed flow is a distinct characteristic of penumbrae that is closely associated with the photometric and magnetic structure of sunspots.

Aims. We analyse the properties of an anomalous flow in the photosphere in a sunspot penumbra and compare it with those of the regular Evershed flow.

Methods. High-resolution spectropolarimetric observations of active region NOAA 11271 were obtained with the spectro-polarimeter (SP) on board Hinode. We used the magnetic field properties derived by a Milne-Eddington inversion. In addition, we used Ca II H images obtained by the broad-band filter instrument to study the low chromospheric response to the photospheric structure and Dopplergrams from the Helioseismic and Magnetic Imager to follow the evolution of the photospheric flows.

Results. We detect a blueshifted feature that appeared on the limb-side penumbra of a sunspot and that was present intermittently during the next two hours. It exhibited a maximum blueshift of $1.6 \mathrm{~km} \mathrm{~s}^{-1}$, an area of $5.2 \operatorname{arcsec}^{2}$, and a maximum uninterrupted lifetime of $1 \mathrm{~h}$. The blueshifted feature, when present, lies parallel to redshifts. Both blue- and redshifts flank a highly inclined or horizontal magnetic structure that is radially oriented in the penumbra. The low-cadence SP maps reveal changes in size, radial position in the penumbra, and line-of-sight (LOS) velocity of the blueshifted feature, from one scan to the next. There was an increase of nearly $500 \mathrm{G}$ in the field strength with the onset of the blueshifts, particularly when the LOS velocity in the feature exceeded $1.5 \mathrm{~km} \mathrm{~s}^{-1}$. There was only a marginal reduction in the field inclination of about $10^{\circ}$ with the increase in blueshifts. In the chromosphere, intense, arc-shaped brightenings were observed close to the location of the photospheric blueshifts, which extend from the edge of the umbral core to the penumbra-quiet Sun boundary. The intensity of these brightenings exceeds the background intensity by $30 \%$ to $65 \%$ with the strongest and largest brightenings observed about $30 \mathrm{~min}$ after the strongest blueshifts were detected at the photosphere. The close spatial proximity of the two phenomenon strongly suggests a causal relationship.

Conclusions. The blueshifted feature represents plasma motion that could be related to a magnetic structure that rises in the solar atmosphere and subsequently reconnects with the ambient chromospheric magnetic field of the sunspot or an inverse Evershed flow, which would be unique in the photosphere. This transient phenomena is presumably related to the dynamic stability of the sunspot because the corresponding umbral core separated two days later at the location of the blueshifts and fragmented subsequently.
\end{abstract}

Key words. Sun: magnetic fields - Sun: photosphere - Sun: chromosphere - techniques: photometric - techniques: polarimetric sunspots

\section{Introduction}

The Evershed flow (EF; Evershed 1909) is a characteristic property of sunspot penumbrae that has been studied extensively for several years (Ichimoto 1987; Sanchez Almeida \& Lites 1992; Rimmele 1994; Balthasar et al. 1996; Westendorp Plaza et al. 1997; Mathew et al. 2003; Bellot Rubio et al. 2004; Beck 2008, to mention only a few). The EF comprises a nearly horizontal and radial outflow of plasma. Consequently, the centre- and limb-side penumbra are blue- and redshifted respectively, when the sunspot is located off the disk centre. In the upper solar atmosphere the direction of the EF reverses and flows into the sunspot and as such is referred to as the reverse/inverse EF (St. John 1913). However, the formation of a penumbra is sometimes associated with flows that have an opposite sign to the EF in the photosphere (Schlichenmaier et al. 2012) and can even be supersonic (Louis et al. 2013). Bellot Rubio et al. (2008) show an example of an oppositely directed EF associated with decaying penumbral filaments.

Penumbrae can also harbour the EF and a counter-EF at the photosphere, as in the case of $\delta$-spots where convergent flows slip past one another and return to the solar interior (Lites et al. 2002) or in regular sunspots that consist of disjointed penumbrae residing within the primary penumbra (Kleint \& Sainz Dalda 2013). Apart from the EF other anomalous flows are also known to exist in the penumbra. Katsukawa \& Jurčák (2010) report the existence of small downflow patches measuring 0.'5 in size that were sometimes associated with brightenings in the chromosphere. Since these patches were often seen on the centre-side penumbra and had the same polarity as the sunspot, their origin, according to Katsukawa \& Jurčák (2010), appeared to be unrelated to the EF. Louis et al. (2011) discovered large patches of supersonic downflows at or close to the umbra-penumbra boundary of sunspots with lifetimes of more than $14 \mathrm{~h}$ that were accompanied by strong and long-lived chromospheric brightenings.

The physical mechanism responsible for the EF is intimately connected to the photometric, magnetic, and kinematic structures of the penumbra, as well as to its fine structure, which is in itself a highly debated topic (Thomas \& Montesinos 1993; Schlichenmaier et al. 1998; Rimmele \& Marino 2006; Borrero \& Solanki 2008; Puschmann et al. 2010; Joshi et al. 2011; Ruiz Cobo \& Asensio Ramos 2013; Scharmer et al. 2013). The 
EF has been described in terms of a siphon flow (Thomas \& Montesinos 1993), where the outflow is a result of the pressure gradient at the footpoints of a flux tube. On the other hand, the "moving-tube" model of Schlichenmaier et al. (1998) shows that the EF arises from a combination of hot plasma rising at the inner footpoint of the tube and a pressure difference from radiative losses. More recently, Rempel (2011) has demonstrated that the Evershed flow in three-dimensional magneto hydrodynamic simulations can be understood as the convective flow component in the direction of the magnetic field where the penumbral fine structure results from anisotropic magneto-convection. In this paper, we study the evolution of an atypical penumbral flow and compare its properties with the well-known photospheric EF.

\section{Observations}

For this investigation, we utilize spectropolarimetric observations from the Hinode spectropolarimeter (SP, Lites et al. 2001; Ichimoto et al. 2008) for active region (AR) NOAA $11271 \mathrm{ob}-$ served on 2011 August 19. The AR was mapped by the SP in the fast mode from 8:05-10:21 UT resulting in a total of five scans that were acquired at 8:05-8:21 UT, 8:30-8:46 UT, 8:559:11 UT, 9:35-9:51 UT, and 10:05-10:21 UT, respectively. The $\mathrm{SP}$ recorded the four Stokes parameters of the Fe I lines at $630 \mathrm{~nm}$ with a spectral sampling of $21.5 \mathrm{~mA}$, a step width of 0.29 and a spatial sampling of 0.32 along the slit. The field-ofview (FOV) covered by the SP was $75^{\prime \prime} \times 82^{\prime \prime}$. The observations were reduced with the corresponding routines of the solar-soft package (Lites \& Ichimoto 2013) to yield Level-1 data. The AR was located at a heliocentric angle $\Theta$ of $29^{\circ}$. For this analysis, we used Level-2 maps comprising two-dimensional maps of the magnetic field strength, inclination, azimuth, and line-of-sight (LOS) velocity that were obtained from inversions of the Stokes profiles with the MERLIN ${ }^{1}$ (Lites et al. 2007) code. The inclination and azimuth were subsequently transformed to the local reference frame. We use the mean umbral velocity as the zerovelocity reference. All the SP maps were co-aligned with respect to the first scan. The SP observations were complemented with co-temporal broad-band filtergrams in $\mathrm{Ca}$ II $\mathrm{H}$ and $G$-band that had a spatial sampling of 0.1 and a cadence of $2 \mathrm{~min}$.

We also utilized data from the Helioseismic and Magnetic Imager (HMI; Schou et al. 2012), namely, continuum intensity filtergrams with a spatial sampling of 0.5 and a cadence of $12 \mathrm{~min}$ to trace the evolution prior and after the SP observations and Dopplergrams at a cadence of $3 \mathrm{~min}$ to follow the evolution of the LOS velocity between the SP maps.

\section{Results}

\subsection{Blueshifted feature on the limb-side penumbra}

The top lefthand panel of Fig. 1 depicts the leading sunspot in AR 11271, which comprises two prominent light bridges (LBs) that separate the primary umbra into three umbral cores. The MERLIN maps shown in the figure correspond to the last SP scan acquired between 10:05-10:21 UT. Our area of interest clearly shows a blueshifted filamentary structure on the limbside penumbra that lies adjacent and parallel to a redshifted feature (RSF). The blueshifted feature (BSF) has a maximum blueshift of $1.6 \mathrm{~km} \mathrm{~s}^{-1}$ and an area of $5.2 \operatorname{arcsec}^{2}$. The BSF has

\footnotetext{
1 Level-2 maps from MERLIN inversions are provided by the Community Spectro-polarimetric Analysis Center at the following link - http://www. csac.hao.ucar.edu/csac
}


Fig. 1. Leading sunspot in NOAA AR 11271. Top panels, clockwise from top left: $G$-band intensity, LOS velocity, field inclination in the local reference frame, and field strength. The MERLIN maps correspond to the last SP scan acquired from 10:05-10:21 UT. Blue and red colours in the LOS velocity map correspond to blue- and redshifts, respectively. The arrow in the top left panel points to disk centre. Bottom panel: arrows corresponding to the horizontal magnetic field overlaid on the LOS velocity map. The FOV under study is marked by the dashed rectangle in the top panels.

a width and length of $1^{\prime \prime}$ and $5^{\prime \prime}$, respectively. The $G$-band image shows a bright penumbral grain close to the BSF and RSF in the inner penumbra. Both the BSF and RSF extend from the smaller umbral core to the penumbra-quiet Sun (QS) boundary. The BSF is located nearly perpendicular to the line-of-symmetry of the sunspot. In comparison, the LOS velocity in the RSF is about $1 \mathrm{~km} \mathrm{~s}^{-1}$, while the redshifts in the limb-side penumbra are about $1.5 \mathrm{~km} \mathrm{~s}^{-1}$.

The inclination map reveals that the BSF and RSF flank a horizontal elongated structure with the typical inclination in the BSF varying between $95-100^{\circ}$. The RSF has a similar inclination to the BSF, but a few pixels close to the penumbra-QS boundary exhibit opposite polarity. The field strength in the BSF varies from 1500-700 G, decreases with radial distance, and is stronger than in the RSF by about $500 \mathrm{G}$. The bottom panel of Fig. 1 shows the horizontal magnetic field overlaid on the LOS velocity map. The sunspot has a negative polarity, and assuming a smooth azimuth around the spot, we find that the horizontal field is well aligned along the BSF and RSF. As one approaches the smallest umbral core close to the BSF, the horizontal magnetic field is more oblique to the two features. The figure also indicates an inward incursion of the penumbra-QS boundary at the BSF relative to the RSF. 

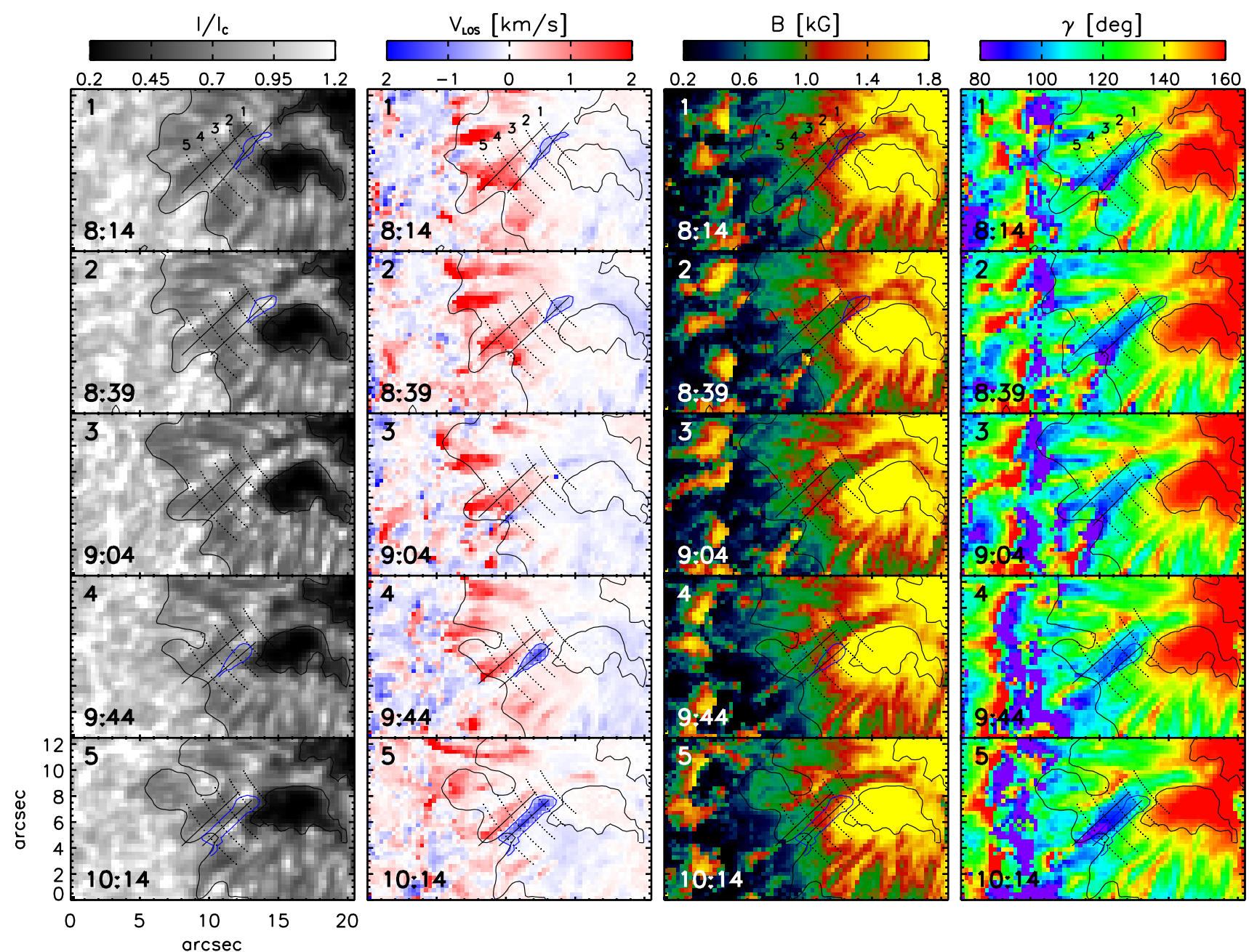

Fig. 2. Temporal evolution of physical parameters. From left to right: $G$-band intensity, LOS velocity, field strength, and inclination. The time indicated in the bottom left corner corresponds to the instant when the SP slit was over the BSF. The thin blue contour outlines the BSF and corresponds to $-0.2 \mathrm{~km} \mathrm{~s}^{-1}$. Panel numbers are shown in the top left corner. Five azimuthal cuts at different radial distances and indexed 1-5 are indicated in panel 1. The plot also shows two radial lines passing through the BSF and RSF.

\subsection{Temporal evolution of blueshifted feature}

The temporal evolution of the BSF and the related physical parameters in the SP maps are shown in Fig. 2 while the corresponding HMI LOS velocity maps are shown in Fig. 5 below. Figure 2 shows the maps from the five SP scans stacked in time from top to bottom. It is evident that the blueshifts at the limb-side penumbra and enclosed in a thin blue contour evolve substantially over a period of $2 \mathrm{~h}$. The LOS velocity map corresponding to panel 3 is nearly devoid of any discernible blueshifts, with the exception of extremely weak blueshifts close to the small umbral core and at the outer penumbral boundary. We defined the central axes of the BSF and RSF in each scan manually by straight lines. These radial lines indicate that the redshifts are present, either behind or alongside the BSF, when the latter is observed but exist even in the latter's absence as indicated in panel 3. Values along the central axes are shown in Fig. 3. Additionally, we laid a series of five azimuthal rings, each radially separated by $1.7^{\prime \prime}$, at a fixed position through the corresponding section of the penumbra. The variation of the various parameters along a few selected azimuthal cuts are shown in Fig. 4. These cuts pass through pixels where the LOS velocity in the BSF was maximum.

Panels 4 and 5 of Fig. 2 show that the end of the blueshifted blob close to the umbra tends to move closer to the inner penumbra with time. A similar feature is also seen in panels 1 and 2 although the blueshifts are weaker than those seen nearly $1 \mathrm{~h}$ later. The maximum blueshifts observed from the scans indicated in panels $1,2,4$, and 5 are $0.5 \mathrm{~km} \mathrm{~s}^{-1}, 0.8 \mathrm{~km} \mathrm{~s}^{-1}$, $1.4 \mathrm{~km} \mathrm{~s}^{-1}$, and $1.6 \mathrm{~km} \mathrm{~s}^{-1}$, respectively. The figure also shows that the radial width of the BSF evolves with time, reaching its maximum during the last SP scan/map. Furthermore, the position of the BSF is closer to the larger umbral core in panels 1 and 2, while it lies farther out in the penumbra about $1 \mathrm{~h}$ later (panels 4 and 5). As stated in Sect. 3.1 and seen in the $G$-band panels of Fig. 2, the penumbra-QS boundary is displaced inwards along the radial line passing through the BSF. A comparison of the field strengths in the red- and blueshifted areas reveals that the latter is stronger by $\approx 500 \mathrm{G}$. The maps of the field inclination indicate that the blueshifts lie on the edge of a highly inclined or even horizontal magnetic structure that persists throughout the period of the SP scans. In panels 1 and 5 the polarity in this filamentary structure close to the penumbra-QS boundary has an opposite sign to the sunspot. The mean field strength and inclination in the BSF over the two-hour duration is about $1100 \mathrm{G}$ and $105^{\circ}$.

Figure 3 shows that the BSF is brighter than the RSF by nearly $10-15 \%$ for most of its radial length. There is an increase in the field strength by about $400 \mathrm{G}$ along the length of the BSF only when the LOS velocity is stronger than $1 \mathrm{~km} \mathrm{~s}^{-1}$, as is 


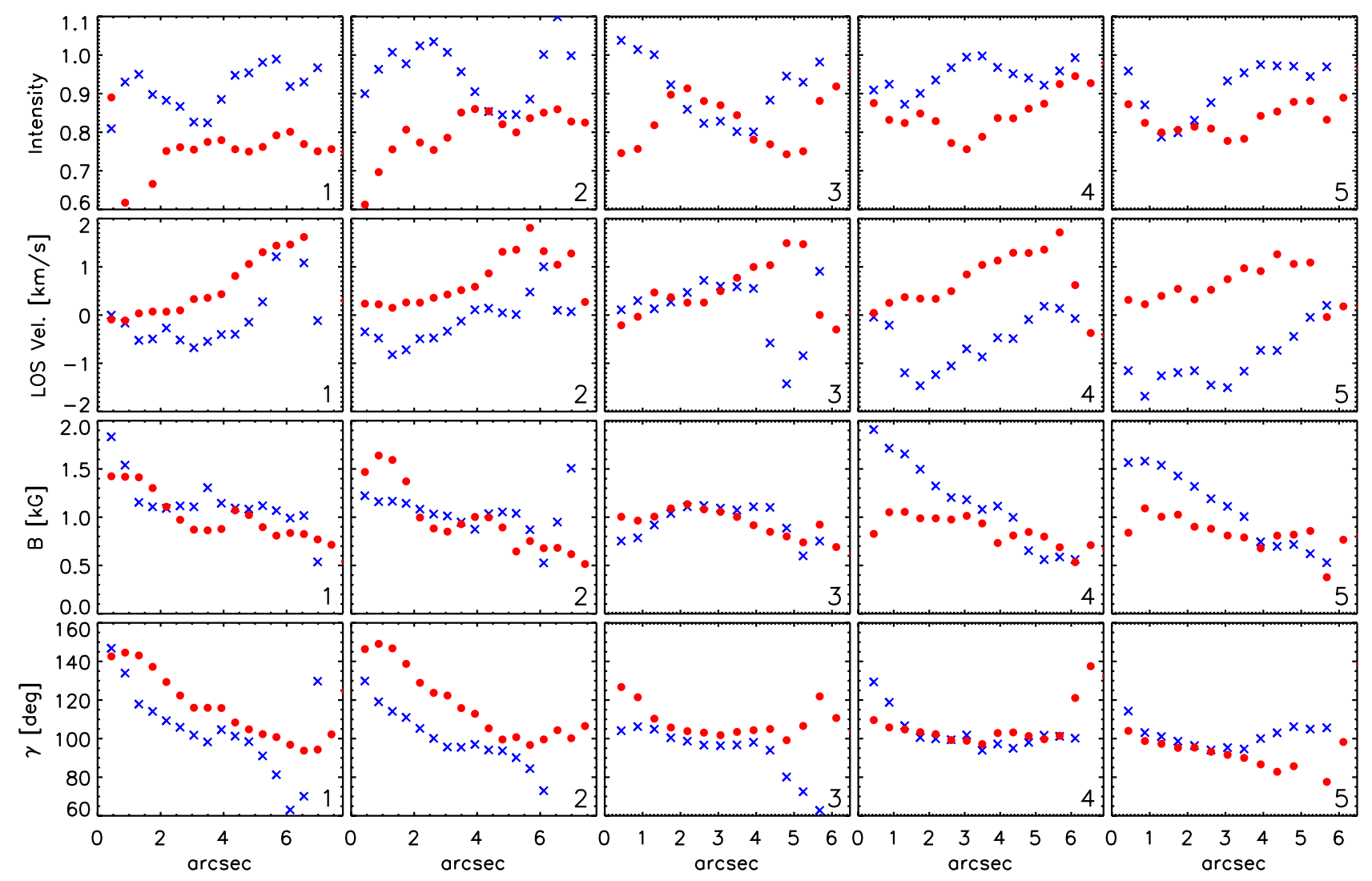

Fig. 3. Variation of parameters along the two radial lines passing through the blue- and redshifted features in Fig. 2. Time increases from left to right as indicated by the number in the bottom right corner, while the blue crosses and red circles correspond to the radial cuts along the blue- and redshifted features, respectively. The left-most point on the $x$-axis corresponds to the upper end of the radial cut.

the case in maps 4 and 5 . In comparison, the field strengths in maps 1 and 2 are about $1100 \mathrm{G}$, when the LOS velocity is less than $1 \mathrm{~km} \mathrm{~s}^{-1}$. The field inclination, on the other hand, reduces by about $10^{\circ}$ with the increase in blueshifts.

Figure 4 shows that the field strength is weaker on the side of the RSF than it is on the side of the BSF. It is also observed that when the blueshifts increase with time, as seen between maps 2 and 4 , the increase in the field strength is around 300-500 G in the BSF. With the exception of the first SP map, the position of the minimum field strength is seen at or near the RSF with values of about $1000 \mathrm{G}$. As stated earlier, the BSF and RSF appear on the edge or side of a nearly horizontal magnetic structure, with the minimum inclination varying between $95^{\circ}-110^{\circ}$. On either side of this horizontal magnetic structure, the field is closer to a vertical orientation by $30^{\circ}$.

\subsection{Recurrence of blueshifts}

The BSF can also be identified in the time sequence of HMI Dopplergrams (Fig. 5). The HMI data reveal that the BSF in the SP maps corresponds to three separate appearances of these features next to the smallest umbral core in the HMI Dopplergrams. The first event starts at 7:40 UT and persists for nearly $1 \mathrm{~h}$ (left column of Fig. 5), while the second event starts around 8:52 UT but only survives for about $20 \mathrm{~min}$. The third event begins at 9:28 UT and has a similar lifetime to the first. It coincides with the duration of the fourth and fifth SP scans. We also find a couple of events during the early part of August 19 , but these are short-lived events with lifetimes of less than 20 min. Similar BSFs are also observed on August 20 when the smaller umbral core started to separate from the sunspot. The HMI Dopplergrams give the impression that the BSFs evolve insitu within the area close to the smaller umbral core. We determined the mean LOS velocity in the BSF using contours (Fig. 5) enclosing blueshifts stronger than $100 \mathrm{~m} \mathrm{~s}^{-1}$. This was done for the time sequence of HMI Dopplergrams for a comparison with the evolution of the intensity in the low chromosphere described in the next section.

\subsection{Chromospheric response}

In this section we analyse the influence of the photospheric blueshifts on the overlying chromosphere. Figure 6 shows a selected set of $\mathrm{Ca}$ II $\mathrm{H}$ filtergrams acquired close to the time interval when the SP slit was over the blueshifted patch. It is evident that the appearance of the BSF in the photosphere affects the chromosphere. The effect is seen in the form of intense arc-shaped brightenings nearly $9^{\prime \prime}$ in length, which extend from the small umbral core to the penumbra-QS boundary. One end of this arc-shaped brightening in the inner/mid penumbra is co-spatial or very close to the BSF. In some cases, there can be isolated bright patches (panel 4) in the chromosphere that later evolve into the arc-shaped structure described above. The brightening is remarkably strong and conspicuous during the last SP map where the blueshifts were also the strongest (panel 12).

We derived a light curve for the region, where the strong brightenings were observed, from the time sequence of the measured relative intensity variation. All pixels inside a hand-drawn contour, whose intensity exceeded the time-averaged intensity inside the same region by $15 \%$ or more, were selected. Their 


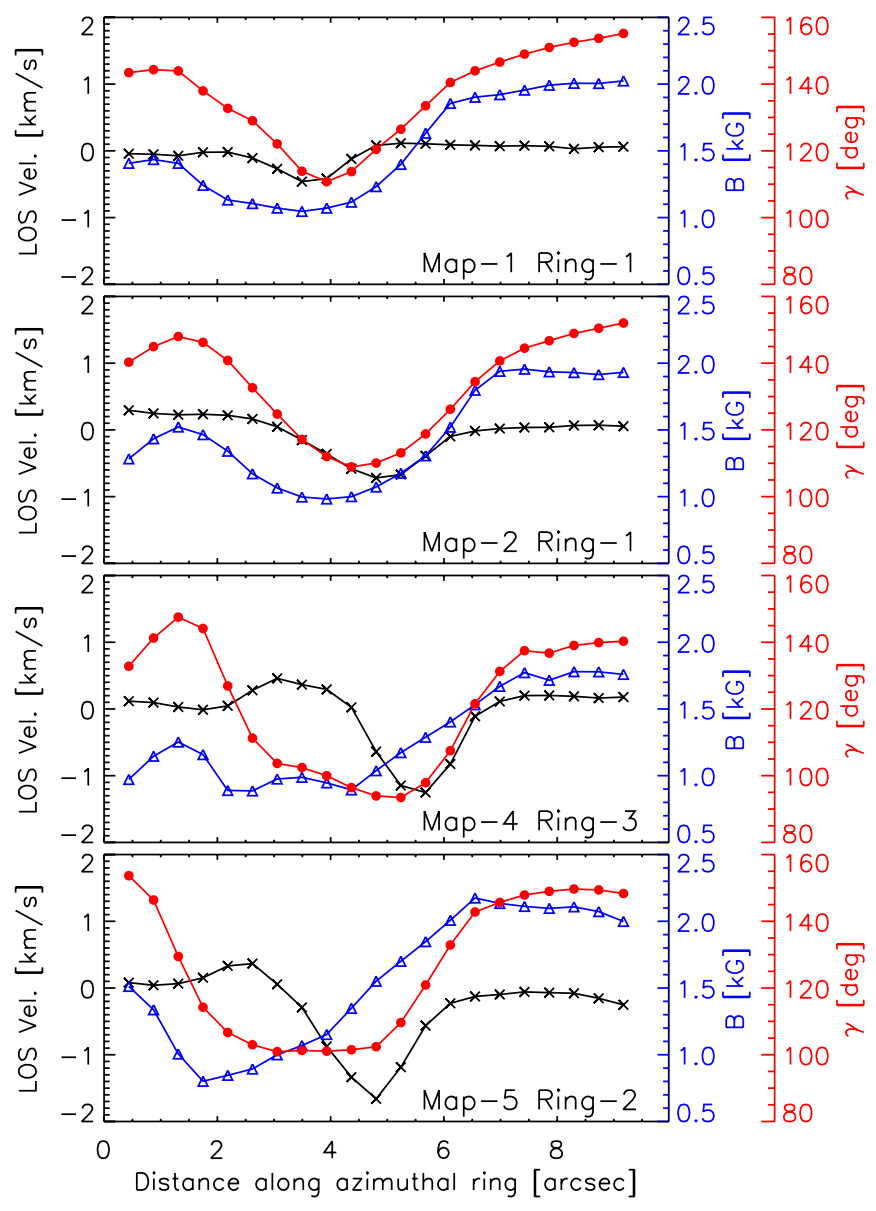

Fig. 4. Variation of parameters along selected azimuthal cuts shown in Fig. 2 for different SP maps. The black line with crosses corresponds to the LOS velocity depicted on the left $y$-axis, while field strength and inclination are shown in blue triangles and red circles, respectively, and indicated on the right $y$-axis. The left-most pixel on the $x$-axis corresponds to the top of the azimuthal cut.

mean intensity as a function of time is shown in Fig. 7. The plot displays the relative intensity with respect to the background and shows that the excess intensity of the chromospheric brightening is between $30 \%$ and $65 \%$. The chromospheric light curve exhibits intermittent peaks during the course of the five SP scans, e.g. at 8:07 UT, 8:41 UT, and 10:27 UT. The intensity peaks occur during the lifetime of the individual blueshifted events that were identified in the HMI Dopplergrams. Furthermore, there appears to be a variable time lag of 10 to $20 \mathrm{~min}$ between the evolution of the photospheric blueshifts and the chromospheric brightenings (Fig. 7). It is also evident from Fig. 7 that the largest blueshift occurs about $30 \mathrm{~min}$ prior to the strongest chromospheric brightening. We note that the two peaks in the chromospheric light curve between SP maps 3 and 4 in Fig. 7 are due to brightenings at the edge of the mask, which covers the penumbra-QS boundary, and they do not exhibit the characteristic arc-shaped structure seen in other filtergrams.

\subsection{Temporal evolution of sunspot}

To relate the above observations with the global sunspot structure, we analyse the evolution of the latter in low-cadence HMI continuum intensity filtergrams spanning a duration of three days beginning on August 18. Figure 8 shows that the leading sunspot has a light bridge separating the umbra into two



Fig. 5. Evolution of photospheric blueshifts seen from a time sequence of HMI dopplergrams. The panels correspond to three events that were detected during the time of the Hinode SP scans. The blue contour outlines the BSF.

nearly equal halves on August 18. In addition, the azimuthal arrangement of the penumbra is disrupted at the location where we observed the blueshifts, nearly $32 \mathrm{~h}$ later. At this location, the penumbra is apparently absent, with granulation extending all the way up to the umbral core. However, the formation of penumbra proceeds rapidly and close to the end of August 18, and the sunspot has a symmetric penumbra, with an additional small light bridge. At the same time, we see that the following spot increases in size. While the Hinode SP observations were made between 8:00-10:00 UT on August 19, the first signs of decay in the leading sunspot appear just $6 \mathrm{~h}$ later, with small penumbral fragments separating from the sunspot. This decay process continues into the early part of August 20 when the entire section of the penumbra close to the smaller light bridge starts to break away. This part of the sunspot eventually separates from the parent spot by 17:00 UT. The parent sunspot also partially recovers its penumbra at the location of fragmentation close to the end of August 20. 




Fig. 6. Chromospheric response to photospheric blueshifts. The four rows correspond to SP scans 1, 2, 4, and 5, respectively. The blue contour represents the BSF at the time when the SP slit was above the feature while the green contour represents the same at other instances of time. See text for description of yellow arrows. The white and black contours correspond to the umbral and penumbral areas, respectively, determined from the $G$-band intensity.

\subsection{Summary}

We have analysed the properties of a blueshifted feature that appeared on the limb-side penumbra of a sunspot, using high resolution spectropolarimetric observations from Hinode. This feature was observed in four out of the five SP scans over a duration of $2 \mathrm{~h}$ and exhibited a maximum blueshift of $1.6 \mathrm{~km} \mathrm{~s}^{-1}$ and an area of $5.2 \operatorname{arcsec}^{2}$. The HMI Dopplergrams reveal that the blueshifts in the SP data were caused by the intermittent appearance of three individual, separate blueshifted patches around the same location during the Hinode observing sequence. The lifetime of two of these events was nearly $1 \mathrm{~h}$, while the third was short-lived and only persisted for about $20 \mathrm{~min}$. Parallel and adjacent to the blueshifted feature are redshifted features that correspond to the regular Evershed flow. The blueshifted feature and the Evershed redshifts flank a highly inclined or nearly horizontal magnetic structure that is radially oriented in the penumbra. The mean field strength and inclination in the blueshifted feature over the two-hour duration are about $1100 \mathrm{G}$ and $105^{\circ}$, respectively. The SP maps represent a snapshot of the blueshifted feature at different epochs as seen from the changes in its size, radial position in the penumbra and LOS velocity. There was an increase of nearly $500 \mathrm{G}$ in the field strength with the onset of the blueshifts, particularly when the LOS velocity in the feature exceeded $1.5 \mathrm{~km} \mathrm{~s}^{-1}$. There was only a marginal reduction in the field inclination of about $10^{\circ}$ with the increase in blueshifts.

In the chromosphere, intense, arc-shaped brightenings were observed close to the location of the photospheric blueshifts, which extend from the edge of the smaller umbral core to the penumbra-QS boundary. The intensity of these brightenings exceed the background intensity by $30 \%$ to $65 \%$ with the strongest and largest brightenings observed during the largest blueshifts. The chromospheric intensity in the neighbourhood of the blueshifts exhibits intermittent peaks during the observing sequence. The strongest chromospheric brightening was observed about $15 \mathrm{~min}$ later to the strongest blueshift detected

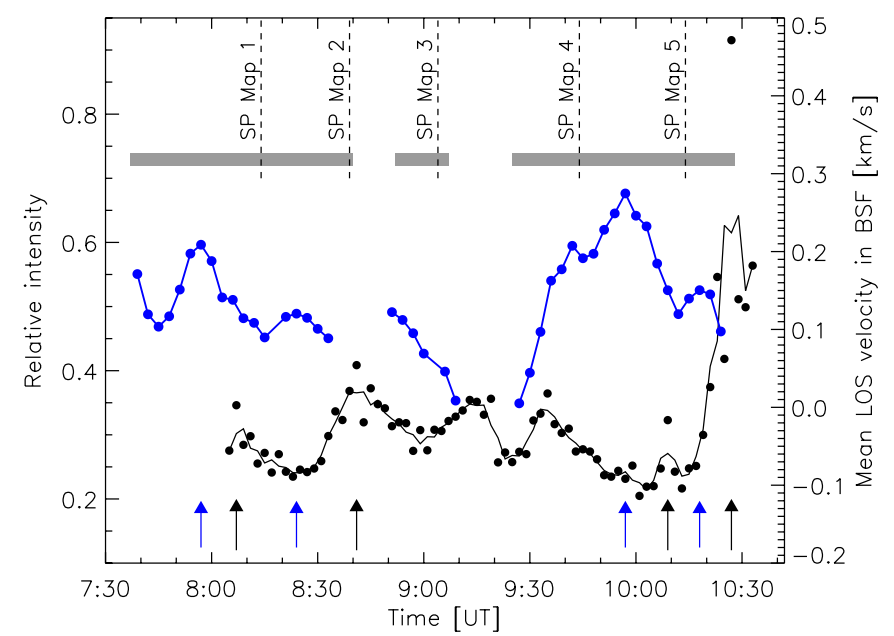

Fig. 7. Chromospheric light curve in the vicinity of the photospheric blueshifts. The solid black line represents a 3-point smoothing to the observed relative intensity (black filled circles). The relative intensity is the excess over the time-averaged background intensity. The grey horizontal bars represent the moments of the BSF's existence. The blue filled circles and solid line correspond to the mean LOS velocity in the BSF estimated from HMI dopplergrams, whose scaling is indicated on the right $y$-axis. The black and blue arrows at the bottom half of the plot indicate local maxima in the chromospheric light curve and photospheric LOS velocities, respectively.

by Hinode and $30 \mathrm{~min}$ after the largest blueshift seen in the HMI data. The other chromospheric brightenings occur about 10-20 min after the photospheric blueshifts reach a maximum value as estimated from HMI Dopplergrams. We speculate that the close spatial and temporal proximity of the two phenomenon is suggestive of a causal relationship.

\section{Discussion}

In the limb-side penumbra one would normally expect only redshifts because of the radial outflow of plasma in the photosphere. The blueshifts detected in the LOS velocity map represent plasma motion towards the observer. One possible scenario is that this plasma motion transports magnetic fields upwards that eventually interact with the overlying sunspot magnetic field. This could lead to reconnection, thereby producing the intense arc-shaped brightenings that occur in close spatial and temporal proximity. From the observed time lag of 10-20 min, magnetic flux moving with speeds of about $1 \mathrm{~km} \mathrm{~s}^{-1}$ would reach low chromospheric heights of 600-1200 km, which would support the above idea. We also do not discard the possibility that the blueshifts could be produced by a radial inflow of plasma. The LOS velocity from a sonic radial inflow of $7 \mathrm{~km} \mathrm{~s}^{-1}$ that makes an angle $(\phi)$ of about $65^{\circ}$ with the line of symmetry is given by $v \sin \Theta \cos \phi$. This yields a value of $1.4 \mathrm{~km} \mathrm{~s}^{-1}$ for $\Theta=29^{\circ}$, which is in good agreement with our observations. This scenario would imply an inverse EF in the photosphere instead of the chromosphere, which would be unique.

Additionally, the long term evolution of the sunspot seen from the HMI intensity filtergrams provides an additional clue that the region was dynamically unstable. Using numerical simulations, Botha et al. (2011) have analysed the influence of nonlinear magneto-convection around magnetic flux tubes. Their simulations involve solving the non-linear resistive MHD equations in a three-dimensional cylindrical domain, for a central cylindrical flux tube surrounded by an annular convection cell. They demonstrate that as convection evolves the annular cell 


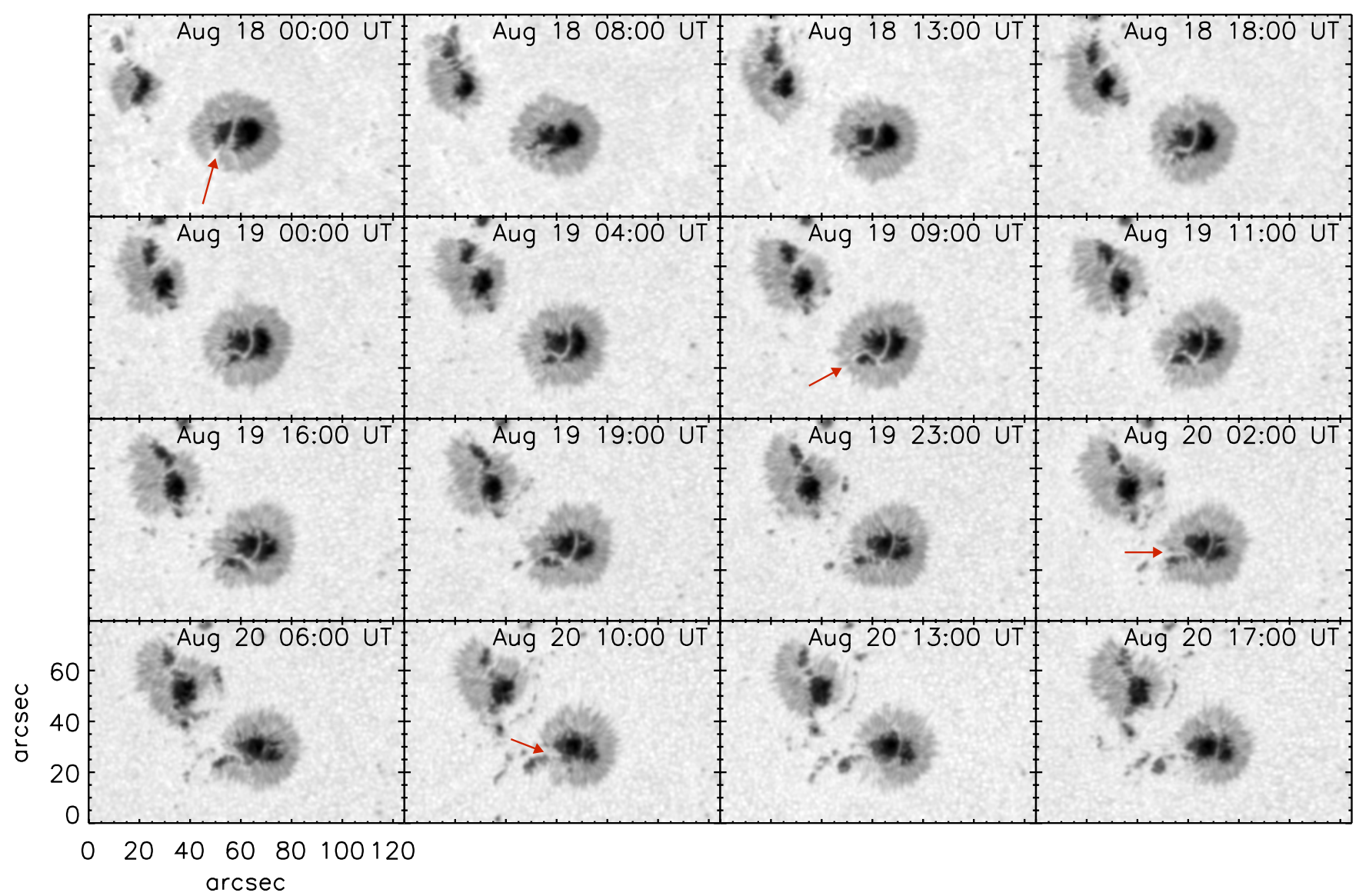

Fig. 8. HMI continuum intensity filtergrams depicting the evolution of the leading sunspot in NOAA AR 11271 over a period of three days starting from 2011 August 18 . Solar north and west point to the top and right respectively.

breaks up in to smaller cells in the azimuthal direction, which allows magnetic flux to slip between these cells away from the central tube through the process of flux erosion. Such a process reduces the magnetic pressure inside the flux tube leading to the growth of convection inside it. The stability of the central tube depends on the convection around it. The simulations also show that magnetic flux can be added to the central tube when flux caught in the surrounding convection is pushed towards it. Such a non-linear interaction between magnetic flux and the surrounding convection could have led to the formation of the penumbral section at the location of the blueshifts in the latter half of August 18.

The blueshifts we have described could be the result of plasma moving towards the umbral core as a result of magnetic flux being pushed and added to the sunspot by the surrounding convective flows. The sunspot also exhibits a weak clockwise rotation, as seen from the HMI images (Fig. 8), that could be associated with the buoyant rise of a magnetic flux rope (Brown et al. 2003; Fang et al. 2012). Such a rotation, albeit small, could play a destabilizing role, in addition to the surrounding convection, and would eventually sever the temporary penumbral attachment of this section of the sunspot. The onset of convective instabilities in rotating cylindrical flux tubes has been described in numerical experiments of Botha et al. (2008). The location of the blueshifted feature coincides well with the line along which the sunspot starts to split one day later while the chromospheric reaction associated with the blueshifts is suggestive of reconnection events. The appearance of the blueshifted feature thus was likely to be the indicator for the development of an instability in the magnetic field configuration at this place.
Our results require the need for high resolution spectropolarimetric observations with good temporal resolution and coverage both in the photosphere and chromosphere so as to determine the physical mechanism responsible for such anomalous flows. Instruments such as the Blue Imaging Solar Spectrometer (BLISS, Puschmann et al. 2013) that has been planned at the $1.5 \mathrm{~m}$ GREGOR telescope (Schmidt et al. 2012), the Interferometric BIdimensional Spectrometer (IBIS; Cavallini 2006), and the CRisp Imaging SpectroPolarimeter (CRISP; Scharmer et al. 2008) will be crucial for such investigations.

\section{Conclusions}

We find an anomalous flow pattern opposite to the regular Evershed flow, i.e. a strong blueshift, in the limb-side penumbra of a sunspot at photospheric heights. Intermittent, arch-shaped brightenings appear in the chromosphere above the feature. The sunspot splits open roughly along the location of the observed blueshift while ejecting one of its umbral cores. We conclude that the anomalous flow in our case very likely indicates a substantial change in the magnetic field topology of the sunspot and is a precursor of the later loss of magnetic flux. Anomalous flow patterns could play a crucial role as indicators of the dissolution and fragmentation of sunspots in their late-life phase.

Acknowledgements. Hinode is a Japanese mission developed and launched by ISAS/JAXA, collaborating with NAOJ as a domestic partner, NASA, and STFC (UK) as international partners. Scientific operation of the Hinode mission is conducted by the Hinode science team organized at ISAS/JAXA. This team mainly consists of scientists from institutes in the partner countries. Support for the post-launch operation is provided by JAXA and NAOJ (Japan), STFC 
(UK), NASA, ESA, and NSC (Norway). Hinode SOT/SP Inversions were conducted at NCAR in the framework of the Community Spectro-polarimtetric Analysis Center (CSAC; http://www.csac.hao.ucar.edu/). HMI data are courtesy of NASA/SDO and the HMI science team. They are provided by the Joint Science Operations Center - Science Data Processing at Stanford University. R.E.L. is grateful for the financial assistance from the German Science Foundation (DFG) under grant DE 787/3-1. We thank the referee for his/her helpful suggestions and comments.

\section{References}

Balthasar, H., Schleicher, H., Bendlin, C., \& Volkmer, R. 1996, A\&A, 315, 603 Beck, C. 2008, A\&A, 480, 825

Bellot Rubio, L. R., Balthasar, H., \& Collados, M. 2004, A\&A, 427, 319

Bellot Rubio, L. R., Tritschler, A., \& Martínez Pillet, V. 2008, ApJ, 676, 698

Borrero, J. M., \& Solanki, S. K. 2008, ApJ, 687, 668

Botha, G. J. J., Busse, F. H., Hurlburt, N. E., \& Rucklidge, A. M. 2008, MNRAS, 387,1445

Botha, G. J. J., Rucklidge, A. M., \& Hurlburt, N. E. 2011, ApJ, 731, 108 Brown, D. S., Nightingale, R. W., Alexander, D., et al. 2003, Sol. Phys., 216, 79 Cavallini, F. 2006, Sol. Phys., 236, 415

Evershed, J. 1909, MNRAS, 69, 454

Fang, F., Manchester, IV, W., Abbett, W. P., \& van der Holst, B. 2012, ApJ, 745, 37

Ichimoto, K. 1987, PASJ, 39, 329

Ichimoto, K., Lites, B., Elmore, D., et al. 2008, Sol. Phys., 249, 233

Joshi, J., Pietarila, A., Hirzberger, J., et al. 2011, ApJ, 734, L18

Katsukawa, Y., \& Jurčák, J. 2010, A\&A, 524, A20

Kleint, L., \& Sainz Dalda, A. 2013, ApJ, 770, 74

Lites, B., Casini, R., Garcia, J., \& Socas-Navarro, H. 2007, Mem. Soc. Astron. It., 78,148
Lites, B. W., \& Ichimoto, K. 2013, Sol. Phys., 283, 601

Lites, B. W., Elmore, D. F., \& Streander, K. V. 2001, in Advanced Solar Polarimetry - Theory, Observation, and Instrumentation, ed. M. Sigwarth, ASP Conf. Ser., 236, 33

Lites, B. W., Socas-Navarro, H., Skumanich, A., \& Shimizu, T. 2002, ApJ, 575, 1131

Louis, R. E., Bellot Rubio, L. R., Mathew, S. K., \& Venkatakrishnan, P. 2011, ApJ, 727, 49

Louis, R. E., Mathew, S. K., Puschmann, K. G., Beck, C., \& Balthasar, H. 2013, A\&A, 552, L7

Mathew, S. K., Lagg, A., Solanki, S. K., et al. 2003, A\&A, 410, 695

Puschmann, K. G., Ruiz Cobo, B., \& Martínez Pillet, V. 2010, ApJ, 720, 1417

Puschmann, K. G., Denker, C., Balthasar, H., et al. 2013, Opt. Eng., 52, 081606

Rempel, M. 2011, ApJ, 729, 5

Rimmele, T. R. 1994, A\&A, 290, 972

Rimmele, T., \& Marino, J. 2006, ApJ, 646, 593

Ruiz Cobo, B., \& Asensio Ramos, A. 2013, A\&A, 549, L4

Sanchez Almeida, J., \& Lites, B. W. 1992, ApJ, 398, 359

Scharmer, G. B., Narayan, G., Hillberg, T., et al. 2008, ApJ, 689, L69

Scharmer, G. B., de la Cruz Rodriguez, J., Sütterlin, P., \& Henriques, V. M. J. 2013, A\&A, 553, A63

Schlichenmaier, R., Jahn, K., \& Schmidt, H. U. 1998, ApJ, 493, L121

Schlichenmaier, R., Rezaei, R., \& González, N. B. 2012, in 4th Hinode Science Meeting: Unsolved Problems and Recent Insights, eds. L. Bellot Rubio, F. Reale, \& M. Carlsson, ASP Conf. Ser., 455, 61

Schmidt, W., von der Lühe, O., Volkmer, R., et al. 2012, Astron. Nachr., 333, 796

Schou, J., Scherrer, P. H., Bush, R. I., et al. 2012, Sol. Phys., 275, 229

St. John, C. E. 1913, ApJ, 37, 322

Thomas, J. H., \& Montesinos, B. 1993, ApJ, 407, 398

Westendorp Plaza, C., del Toro Iniesta, J. C., Ruiz Cobo, B., et al. 1997, Nature, 389,47 